\title{
Changement réel et changement induit Décalage ou perpétuelle recherche pour les zones arides d'Afrique du Nord
}

Veronique Alary ${ }^{1}$

Mohammed El Mourid ${ }^{2}$

${ }^{1}$ Centre de coopération internationale en recherche agronomique pour le développement (Cirad),

Département " Élevage et médecine

vétérinaire tropicale " (Emvt),

Cirad-Emvt TA30/A,

Campus de Baillarguet,

34398 Montpellier cedex 5

France

<veronique.alary@cirad.fr>

2 International Center

for Agricultural Research in the Dry Areas

(ICARDA),

1 , rue des oliviers,

El Manzah 5,

1004Tunis

Tunisie

Regional ProgramTunis

<m.elmourid@cgiar.org>

\begin{abstract}
Résumé
L'histoire des politiques de développement économique et social au Maghreb a fortement marqué l'évolution des systèmes agropastoraux des zones arides. En l'espace de 50 ans, on est passé de systèmes d'élevage pastoraux à des systèmes mixtes combinant différents élevages, la céréaliculture, l'arboriculture et une ou plusieurs activités extra-agricoles. Dans ce contexte, le changement induit par l'innovation technologique a du mal à émerger, notamment lorsque l'innovation s'intéresse à la durabilité de la ressource qui assure aujourd'hui une part minime de l'alimentation du troupeau. Ces innovations induites par la recherche, mais coproduites avec les agents du développement et les éleveurs, se heurtent à des dynamiques sociétales largement orientées vers la modernité des modes de vie, les profits de court terme pour la consommation. Les processus d'introduction de ces technologies se heurtent également à la nouvelle configuration sociale et spatiale des populations agropastorales.
\end{abstract}

Mots clés : changement technologique ; Maghreb ; politique agricole ; système agropastoral ; zone aride.

Thèmes : économie et développement rural ; climat ; systèmes agraires.

\begin{abstract}
Between real change and induced changes - Discrepancy or perpetual research for the arid areas of North Africa

Pastoral systems in the arid zones of the Maghreb have undergone important changes due to economic and social development policies. Within the last 50 years, pastoral systems have virtually gone over to mixed systems that combine different animal activities, cereal crops, olive trees and off-farm activities. In this context, the changes brought about by new technologies are not easily accepted. Difficulties increase for natural resource management technologies implying long-term processess. Moreover, natural resources contribute but little to the animal feeding calendar. These technologies favoured by research but co-produced with the stakeholders (farmers, technicians, developers) come up against social changes largely oriented towards modern ways of life and short-term profits for rapid consumption. The diffusion of new technologies also comes up against the new social and spatial configuration of agro-pastoral populations.
\end{abstract}

Key words: agricultural policies; agropastoral systems; arid zones; Maghreb; technological changes.

Subjects: economy and rural development; climate; farming systems.

i les sociétés pastorales des zones arides du Maghreb ont été soumises aux mêmes règles imposées par les grands courants de la pensée politique (socialisation, puis libéralisation), elles ont subi un double traumatisme lié aux politiques de sédentarisation, puis d'appropriation, parfois non 
contrôlée, des terres communales. Les mouvements successifs de socialisation post-indépendance, puis de libéralisation dans les années 1980, ont fait passer ces exploitations d'un assistanat étatique à une situation de dépendance aux lois du marché. Ces changements largement aidés par l'environnement extérieur ont suscité un ensemble de dynamiques sociales et économiques qui définissent aujourd'hui la société agropastorale.

Dans ce contexte, l'innovation technologique d'abord inscrite dans de grands projets de développement basés sur la modernisation, puis atomisée aux échelles locales, a eu du mal à passer au stade de progrès et de changements techniques dans les exploitations des zones arides. Aujourd'hui, les options techniques promues par la recherche et le développement s'appuient sur la reforestation, la lutte contre la désertification, et le choix de variétés résistantes. Si ces solutions sont plus raisonnables d'un point de vue agronomique et écologique, elles s'éloignent des besoins des populations rurales avides de modernité, de productivité, de rentabilité à court terme. Ainsi, le changement souhaité a du mal à se réaliser ; il est submergé par les changements spontanés et réels, imposés par l'environnement politique et social.

Ainsi, à partir de l'analyse des changements réels largement aidés par l'histoire des politiques qui a façonné le milieu, y compris les hommes, on se propose de rechercher les raisons de résistance à l'innovation, changement souhaité et aussi voulu par l'extérieur.

\section{Changements réels}

Si les grands courants de la pensée politique d'après-guerre des pays en développement (socialisation, collectivisation, autonomie) ont fortement influencé le cadre et la philosophie de la construction des politiques agricoles au Maghreb, à maintes reprises, les particularismes liés à l'histoire ou la société vont entrainer des différences notables dans la mise en œuvre des politiques comme des effets contrastés dans chacun des pays. Toutefois, on peut dégager des tendances communes entre les trois pays étudiés (Algérie, Maroc et Tunisie) qui s'inscrivent dans le changement réel des sociétés pastorales.

\section{Le contexte politique comme facteur de changement exogène}

Le fonctionnement actuel des systèmes pastoraux des zones arides au Maghreb est largement ancré dans le système sociétal et son évolution mais aussi son environnement politique et économique. Or cet environnement politique a été marqué par une volonté de contrôle social et territorial des zones arides depuis la colonisation et le souci postindépendance de construire une unité nationale. Ainsi, les premières réformes engagées dans les zones arides ont visé à détruire les formes précapitalistes d'organisation (tribus, notables) et à les remplacer par une administration moderne (Belhedi, 1989). Il s'agirait pour l'État de contrecarrer le contre-pouvoir traditionnel comme de maîtriser les formes de mobilité et d'accumulation qui lui échappent. Durant cette phase de contrôle territorial, le seul bien commun - le parcours - sur lequel les sociétés pastorales s'étaient structurées et organisées socialement fait l'objet non seulement de nombreuses réformes mais aussi de dynamiques d'appropriation, le plus souvent non contrôlées (Abaab et al., 1995; Bourbouze et Gibon, 1995).

Le deuxième traumatisme s'inscrit dans l'idéologie socialisante des années postindépendance basée sur la nationalisation des moyens de production, y compris la terre et le cheptel. Dans la collectivisation, le seul bien des pasteurs - les animaux - devient propriété collective. Perte du tissu social liée aux réformes agraires et perte de patrimoine, ces réformes ont profondément remodelé la répartition du cheptel avec une concentration du cheptel chez les gros éleveurs, notamment au Maroc, et la formation d'un salariat chez les petits éleveurs, notamment en Algérie (Guillermo, 1990).

Dans la phase d'appropriation foncière, les petits éleveurs s'installent sur des lopins de terre et développent un système mixte basé sur l'élevage de petits ruminants et la céréaliculture. Les années 1970 et 1980 voient le développement de vastes programmes de lutte contre la sécheresse alors que le reste des zones connaît un semblant de révolution verte grâce au développement de grands projets d'irrigation. Par la distribution gratuite ou à bas prix des aliments pour le bétail, ces politiques visent à éviter la décapitalisation (vente des animaux) en période de sécheresse. Les conséquences de ces politiques sont bien connues : maintien d'un troupeau important sur des ressources épuisées par les sécheresses successives, dégradation des ressources naturelles, et donc plus forte dépendance au marché. Ce phénomène s'aggrave avec le déplacement de grande ampleur de l'aliment pour bétail par camion jusqu'au fin fond des steppes. Aujourd'hui, ces sociétés s'inscrivent dans une dynamique rythmée par la succession des sécheresses et l'attente des soutiens et s'opposent en apparence au dynamisme économique des sociétés rurales des zones irriguées qui privilégient dès qu'elles le peuvent un modèle productif à l'exportation (marầchage, oliveraie). Pourtant les zones arides et semi-arides représentent près des deux tiers du territoire et $22 \%$ de la population rurale au Maghreb.

La période d'ajustement des années 19801990 se caractérise par un abandon progressif des principaux tenants des politiques autocentrées, axées sur l'autonomie nationale, notamment en Algérie, et des politiques de collectivisation des moyens de production. La revalorisation des prix agricoles est réalisée successivement par le relèvement des prix administrés des produits de base (céréales, olive à huile, viande ovine et bovine), puis par la libéralisation des prix, avec une diminution progressive des subventions sur les intrants. Mais cette libéralisation reste toutefois partielle. Dans les trois pays, le secteur céréalier reste étroitement contrôlé par l'État (Alary et El Mourid, 2005). Si les gouvernements des trois pays abandonnent les subventions sur la viande, pour des questions de paix sociale, la Tunisie comme le Maroc maintiennent certaines fourchettes de prix, notamment durant les périodes de fêtes religieuses (Ramadan, Aïd-El-Kébir), et les importations restent fortement contrôlées par l'État dans les trois pays du Maghreb. Mais si les données officielles pour les trois pays enregistrent des importations de petits ruminants (vivant ou viande) quasiment nulles, près de 1,8 million d'ovins passeraient les frontières entre l'Algérie et la Tunisie ou l'Algérie et le Maroc, sans compter les flux d'animaux en provenance du sud Sahara. Cette histoire des politiques de développement au Maghreb a fortement marqué l'évolution des systèmes agropastoraux des zones arides. 


\section{Changement des systèmes agropastoraux... Fragilisation de l'activité d'élevage}

Jusqu'aux années 1960, les systèmes traditionnels des zones arides du Maghreb étaient essentiellement basés sur une activité d'élevage dominante conduite sur un mode nomadique ou agropastoral avec une transhumance saisonnière des zones pastorales vers les zones agricoles, notamment durant les périodes postrécoltes. Ces systèmes se caractérisaient par une forte dépendance aux conditions climatiques et donc aux ressources pastorales disponibles qui assuraient près de $65 \%$ des besoins alimentaires du cheptel jusqu'au début du $\mathrm{xx}^{\mathrm{e}}$ siècle (Nefzaoui et al., 2002) et étaient généralement gérées collectivement. Les principaux tenants de ces systèmes étaient le nomadisme, des troupeaux multiespèces de races rustiques, une gestion et exploitation du troupeau à moyen ou long terme avec des réformes tardives.

La réduction de la part des ressources pastorales dans la couverture des besoins du troupeau va entraîner une forte dépendance des éleveurs envers les ressources produites sur l'exploitation ou achetées sur le marché. Et l'introduction encouragée de l'arboriculture et des cultures arbustives fourragères dans les systèmes agropastoraux va favoriser le mouvement de privatisation et de fermeture de l'espace pastoral. La production des céréales s'inscrit aussi progressivement dans la gestion quotidienne de l'alimentation du troupeau alors que le stock céréalier pour la sécheresse s'amenuise.

Parallèlement, les systèmes d'élevage se simplifient structurellement. Dans les milieux agropastoraux, une majorité des exploitations ont abandonné l'élevage caprin, considéré comme trop difficile à maittriser dans un espace qui se ferme et dont une partie est occupée par les cultures. En outre, cet élevage demande du temps de surveillance alors que la maind'œuvre familiale s'amenuise avec la diversification des activités en dehors de la sphère familiale et villageoise. Si les naissances restent toujours réparties sur l'année, ce n'est plus pour les mêmes raisons. Avec une population rurale soit âgée soit féminine, la répartition des agnelages permet d'éviter les périodes de pointe de travail. En revanche, du fait de la généralisation de la complémentation en période sèche et de la recherche de réduction des dépenses d'aliments, l'âge de la réforme tend à se réduire. On voit même se développer dans les zones semi-arides des élevages d'engraissement en bergerie sans brebis. Cette activité a connu un succès important en Tunisie centrale à la suite de la dernière grande sécheresse (1998-2002).

Ces changements s'inscrivent donc dans une dynamique "plurispatiale " et "multiactive " des ménages ruraux des zones arides et semi-arides, que l'on retrouve dans nombre de régions des pays du Sud (Lesourd, 1997). Avec le développement de l'industrie et le mouvement d'urbanisation généralisé dans les années 19701980, l'emploi non agricole, et donc la multiactivité, s'inscrit dans la gestion globale de l'exploitation (Elloumi, 1993). Cela se traduit par un réinvestissement des flux monétaires extérieurs vers l'agriculture familiale et surtout une réallocation de la main-d'œuvre familiale avec une féminisation croissante de l'activité d'élevage alors que les hommes s'emploient à l'extérieur du monde rural, et plus généralement de leur région. Cependant, depuis la crise économique de la fin des années 1990, on assiste à un ralentissement, voire une inversion, des flux de main-d'œuvre dans certaines zones, mais dont on maitrise peu l'ampleur tant les dynamiques sont devenues familiales et non plus communautaires (Sandron, 1997).

Ainsi, le mouvement de dépendance accéléré au marché durant la moitié de la fin $\mathrm{du} \mathrm{xx}^{\mathrm{e}}$ siècle et la réduction récente des opportunités d'emploi à l'extérieur favorisent une gestion de très court terme de l'exploitation. Les stocks d'aliments suffisent à peine à couvrir les besoins annuels du troupeau et la complémentation est devenue un élément structurel même en bonne année. Seuls les gros éleveurs qui possèdent suffisamment de terres arrivent à satisfaire les besoins du troupeau en bonne année. Cette dépendance au marché favorise aujourd'hui le développement de comportements spéculatifs, notamment dans les zones céréalières. À côté, la reconstitution des troupeaux n'excède pas en moyenne une trentaine d'animaux de reproduction afin d'éviter les pertes massives et de limiter les effets des comportements spéculatifs sur les denrées animales notamment l'orge, le son et la paille durant les sécheresses.

On assiste aussi à une certaine spécialisation géographique des producteurs dans la filière animale. En Tunisie, suite aux cinq dernières années de sécheresse (1998-2002), l'activité d'élevage se spatialise avec une fonction de naisseur dans le Nord - région moins affectée par les sécheresses - et une fonction d'engraisseur dans les régions du Centre et du Sud. Cependant, suite aux deux bonnes années climatiques qui ont suivi (20032004), les éleveurs du Centre et du Centre-Sud se sont relancés dans une stratégie de capitalisation dans le cheptel ovin par manque de liquidité et de capital pour acheter les agneaux à l'engraissement. Au Maroc comme en Tunisie, se développe dans les zones favorables une véritable activité de constitution de stocks de paille et de foin dans l'attente d'une sécheresse. Ainsi, au déplacement des animaux, s'est substitué le déplacement des fourrages avec un risque spéculatif non négligeable lié en partie à la libéralisation des prix des intrants sur les marchés. Les systèmes de production qui en résultent sont donc le fruit d'adaptation de pratiques liée aux changements extérieurs (notamment de nature politique), mais les nouveaux comportements qui se dessinent ont d'indéniables conséquences sur l'organisation sociale des ménages comme des communautés.

\section{Recomposition des sociétés}

Les communautés d'éleveurs agriculteurs des zones arides sont généralement composées d'anciennes familles nomades. La plus forte vague de sédentarisation date des décennies 1950 et 1960, ce qui explique qu'on ait à faire principalement à des néopaysans. Il s'agit d'un enracinement relativement récent dans un genre de vie sédentaire. Cela se traduit par un habitat relativement dispersé et des pratiques culturales très extensives.

Que ce soit du temps de la colonisation ou de la période post-indépendance, la substitution de l'État à la communauté a été l'un des enjeux politiques majeurs pour le contrôle des sociétés pastorales, l'objectif étant d'amenuiser le pouvoir des autorités traditionnelles et de désolidariser la communauté. Mais le passage du communautarisme à l'individualisme a créé, ravivé, ou maintenu, de fortes tensions entre les fractions dominantes et les fractions dominées dans la régulation de la gestion du territoire qui incombait jadis à la communauté. En outre, les individus se sont vus forcés d'appartenir à plusieurs configurations socioculturelles, qu'il s'agisse du parti politique, de l'administration ou de la fraction d'origine. Le résultat est à la fois un renforcement des 
réseaux sociaux plus restreints mais aussi l'émergence de comportements individuels au détriment d'une gestion coordonnée. Outre le processus de réforme agraire avec son corollaire de sédentarisation, l'ensemble des réformes institutionnelles et structurelles avec leur pendant de plus d'individuation a aussi profondément affecté le référentiel temps et espace des individus avec des horaires et des calendriers nouveaux comme des unités administratives. Cette individuation donne plus de latitude d'expression mais aussi de possibilités de transgression.

La salarisation des éleveurs, notamment en Algérie, et le développement de la pluriactivité vont aussi changer les mentalités, les modes de consommation, la vie quotidienne, les objectifs poursuivis, avec une recherche continue des opportunités extérieures à la communauté. La relocalisation du troupeau autour de l'exploitation entraîne aussi un changement des modes d'exploitation comme des modes de vie. Si la transhumance était l'affaire des hommes, la sédentarisation entraîne une plus grande implication des femmes dans la gestion du troupeau, renforcée par les départs fréquents des hommes actifs à la recherche de revenus complémentaires en ville.

Ainsi, si les nouvelles unités "ménage" conservent leur complexité par rapport au modèle pastoral (multiples unités de résidence), les mécanismes de coordination des agents au sein des unités domestiques ont changé avec la multiplication des activités : agriculture, élevage, activités extra-agricoles. Cette multiplication des activités et des lieux d'exercice accroît les asymétries informationnelles avec des risques d'opportunisme dans la relation d'échange: multiplication des règles ou des "nœuds" de contrats (Requier-Desjardins, 1994) portant sur les transactions liées (travail, approvisionnement, vente, mandat). Mais elle renforce aussi des liens dans des réseaux plus étroits. En milieu rural, on assiste à des solidarités plus fortes entre ménages au sein de familles élargies où les hommes restés au village (souvent les personnes âgées) doivent veiller sur les ménages où les femmes se retrouvent seules. En milieu urbain, de nouveaux réseaux émergent pour les hommes.

Au-delà de cette multiplication des activités qui tend à marginaliser l'activité d'élevage, une certaine représentation collective de l'élevage perdure, notamment à travers la tradition (dot, mariage, naissance) et la religion, avec un système d'attente réciproque des individus les uns par rapport aux autres qui forment des dispositifs intermédiaires non formalisés. Ces structures fonctionnent bien à l'échelle de la famille élargie, notamment dans la circulation de l'information sur les prix. Mais ces structures sociales excèdent rarement la famille élargie et sont le plus souvent basées sur la réputation, elle-même fondée sur la réussite sociale et économique.

Ainsi, de nouveaux modèles se créent résultant de l'héritage historique, mais adaptés, remodelés à la société maghrébine moderne. La communauté ou la tribu qui entretenait par le biais de la gestion des biens communs des règles d'entente se voit segmenter en un ensemble de familles élargies, parfois en conflit, et les pouvoirs traditionnels supplantés par les pouvoirs administratifs et politiques. Et pourtant les pouvoirs traditionnels subsistent... La famille élargie est devenue le principal réseau où circulent l'information et les idées et où existe l'entraide. Mais c'est à l'échelle de la famille nucléaire que s'opèrent le plus souvent le contrôle social et économique et les décisions de production. Ainsi, se dégage un mélange subtil d'individuel et de collectif. Ces communautés ont l'apparence de sociétés soumises, partagées entre un système traditionnel imposé et des aspirations à la modernité. Cet écart pourrait bien expliquer les difficultés de changements techniques.

\section{Changements induits (innovation extérieure) dans le changement réel}

L'innovation comme moyen d'accélération du changement pose directement la question des facteurs de résistance ou d'accélération du processus d'adoption. Quelles sont les logiques qui soustendent l'adoption ou non de l'innovation? L'expérience montre que l'innovation peut être refusée pendant des décennies et adoptée à un moment donné de l'histoire des sociétés. Cette adoption peut se faire spontanément sans le recours à des politiques ou mesures d'accompagnement incitatives ou être largement induite par l'environnement. Ces facteurs environnementaux sont d'ordre politique, économique, social, mais aussi naturel. Une sécheresse qui dure peut conduire à reconsidérer son système pour faire face aux besoins courants. Mais la réponse à la sécheresse va dépendre des mesures politiques comme des autres opportunités économiques. Les écarts de réponses entre agents à l'intérieur d'une communauté soumise aux mêmes conditions extérieures interrogent aussi les facteurs sociaux.

\section{Histoire du changement technologique}

L'innovation interventionniste des années 1970-1980 axée sur le progrès technique et les performances agronomiques a quelque part sous-estimé la place du social dans les comportements et surestimé la rationalité économique (Chauveau, 1999). Durant cette période, la recherche comme les services publics de vulgarisation et de soutien à l'agriculture au Maghreb s'organisent autour de grands programmes définis par produits et pris en charge par des instituts spécialisés (Alary et El Mourid, 2002). Les principaux efforts se concentrent sur la sélection variétale, l'irrigation, la mécanisation et l'intensification par la fertilisation et les traitements chimiques. Ces améliorations concernent principalement les grandes cultures (céréales, cultures sucrières), puis le maraîchage et l'arboriculture pour l'exportation. Dans le domaine des productions animales, les efforts se portent sur l'intensification fourragère et l'amélioration du potentiel génétique. Cependant, ces efforts de recherche inscrits dans l'idéologie dominante de modernisation ont souvent négligé, voire ignoré, les particularités des systèmes des zones arides, fortement dépendants des conditions climatiques et des ressources naturelles.

Dans les années 1980, l'action des pouvoirs publics se porte alors sur le développement de programmes de recherche pluridisciplinaires intégrés au développement. Pour répondre aux besoins spécifiques des zones arides, est créé le Centre aridoculture de Settat au Maroc qui s'appuie sur une recherche système et action et une recherche de partenariat, notamment avec des universités américaines. C'est l'heure de l'ouverture. Dès 1976, est créé l'Institut des zones arides (Ira) en Tunisie. En Algérie, il faut attendre l'ouverture de la fin des années 1990 avec le développement du Plan national de développement agricole (PNDA) en 
2000 et la création au sein de la recherche scientifique du Centre de recherche scientifique et technique pour les régions arides (CRSTRA) à Biskra

Mais ces projets ne vont pas connaître tous les succès attendus. En outre, l'application de nouvelles techniques comme les variétés plus productives ou le matériel génétique animal donne des résultats très contrastés, avec des coûts environnementaux élevés (déperdition d'espace forestier et de parcours, utilisation de la mécanisation sur des sols fragiles) et des coûts sociaux (perte d'un patrimoine génétique adapté aux conditions arides, déstructuration des actions collectives avec des incitations individuelles). Dans certaines zones, ces techniques n'ont pu voir le jour faute d'infrastructures suffisantes et du faible accès au crédit. En outre, cette recherche réalisée en partie en station a souvent ignoré le savoir-faire des acteurs. La principale conséquence dans les milieux où ces techniques se sont développées est l'amenuisement des actions ou pratiques traditionnelles qui étaient garantes d'un équilibre entre la communauté et leur milieu. Ainsi, on peut se poser la question de la pertinence économique des innovations qui s'inscrivent dans une vision très unifiée du développement basée sur la recherche de productivité mais avec des instruments uniques.

La combinaison d'une approche système, d'un diagnostic agroécologique et socioéconomique et des outils de simulation offrent de nouvelles perspectives pour raisonner et adapter les options technologiques en zone aride (El Mourid et al, 1996a ; El Mourid et al., 1996b). Il faut donc attendre les années 1990 pour voir émerger des recherches transversales. Elles s'inscrivent dans une prise de conscience collective de la place de ces systèmes dans l'économie nationale: emploi, risque de désertification, problème de saturation urbaine, production de l'agneau de l'Aïd-El-Kébir. C'est ainsi que les pratiques locales sont réhabilitées et que la recherche s'oriente vers le développement de technologies dites adaptées ou appropriées à ces milieux. Par "adaptées " ou "appropriées ", les chercheurs entendent des technologies qui ont été plus ou moins choisies par la population en fonction de ses contraintes et de ses atouts et des technologies qui respectent un environnement fragile. Les techniques mélangent souvent progrès et tradition comme les plantations de cactus. D'autres se basent sur le développement de techniques à moindre coût comme les blocs alimentaires (largement réhabilités en Irak durant la dernière décennie d'embargo) (Shideed et al., 2005). À côté persistent des technologies dites "modernistes" comme la sélection variétale.

Dans les faits, les résultats d'adoption restent mitigés et varient bien souvent avec les conditions climatiques ou les soutiens politiques. S'ils s'inscrivent dans une évolution nécessaire largement promue par les nouvelles exigences sociétales en termes de durabilité environnementale, il est difficile de parler de révolution en tant que processus nouveau de production assurant la reproduction des systèmes et dans lequel les populations seraient parties prenantes et motrices. Si ces technologies relèvent bien du mouvement de la "révolution doublement verte " avec la prise en compte des effets sur l'environnement, cette révolution qui s'inscrit dans le temps est-elle possible dans une société jeune en quête de modernité à court terme? En outre, ces technologies sont généralement coûteuses, même si elles s'appuient sur des techniques à faible coût car elles nécessitent un processus de vulgarisation long et rapproché, des aides pour supplanter des techniques moins productives et des efforts importants d'organisation collective.

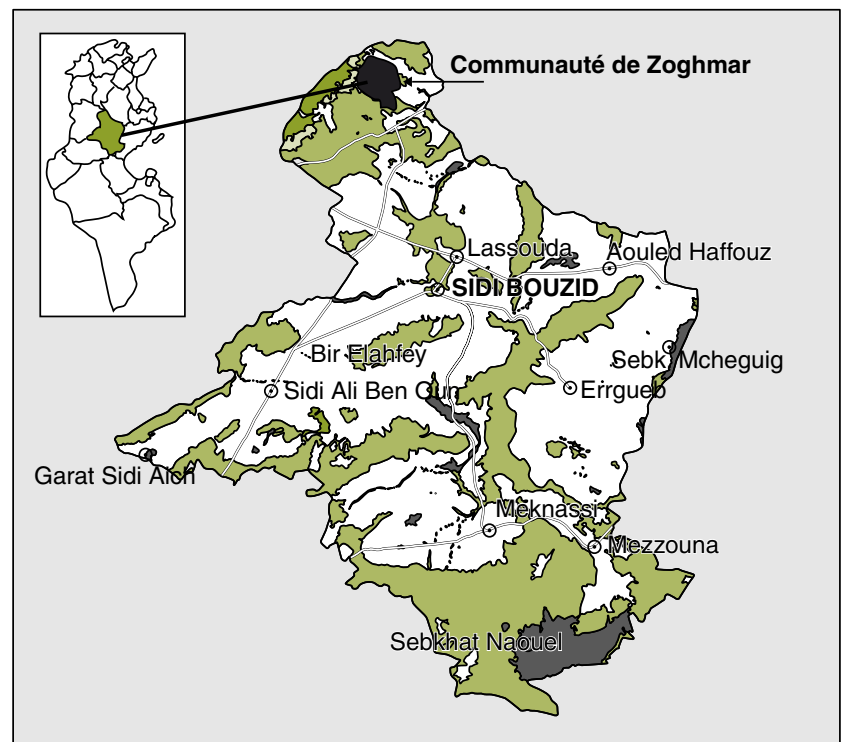

Figure 1. Localisation de la communauté de Zoghmar dans le gouvernorat de Sidi Bouzid (Tunisie centrale).

Figure 1. Localisation of the Zoghmar Community in the Sidi Bouzid Governorate (Central Tunisia). Source : Direction générale des forêts, Tunisie, 1995.

\section{Exemples de différentiation d'adoption d'innovations durables}

L'analyse des taux d'adoption pour la technologie du cactus inerme intercalaire dans une communauté de la zone semiaride de la Tunisie Centrale (communauté de Zoghmar) montre des écarts importants d'adoption selon les systèmes d'exploitation familiale, identifiés à partir de leur structure et mode de fonctionnement (figure 1, tableau 1).

Ainsi, si la communauté agropastorale connaît un taux d'adoption de près de 30,6\% (sur une population de 317 ménages) (Elloumi et al., 2005), les petites exploitations familiales qui représentent près des deux tiers de la population enregistrent un taux voisin de $18 \%$.

Par le biais de la méthode de contingence, nous avons tenté de cerner les raisons des choix d'adoption ou de nonadoption. En d'autres termes, il s'agissait de déterminer à quel niveau de soutien public les éleveurs seraient prêts à adopter la technologie (Alary, 2006) (figure 2). Les résultats montrent que les éleveurs seraient prêts à implanter plus de la moitié du potentiel des surfaces uniquement si l'Office de l'élevage et du pâturage (OEP), office public en charge du développement de l'élevage pastoral en Tunisie, assurait la distribution des raquettes ou s'il finançait le rachat des raquettes 
Tableau 1. Présentation des six types d'exploitation dans la communauté de Zoghmar (Tunisie centrale) et taux d'adoption de la technologie du cactus inerme intercalaire par type (en \%)

Table 1. Description of the six farm types in the Zoghmar community (Central Tunisia) and rate of adoption of the spineless cactus technology in alley-cropping for each farm type (in \%).

\begin{tabular}{|c|c|c|c|c|c|}
\hline $\begin{array}{c}\text { Systèmes } \\
\text { d'exploitation } d^{\prime}\end{array}$ & $\begin{array}{c}\text { Nombre } \\
\text { d'exploitations } \\
\text { par } \\
\text { système }\end{array}$ & $\begin{array}{c}\text { Surface } \\
\text { moyenne } \\
\text { agricole totale } \\
\text { (ha) }\end{array}$ & $\begin{array}{c}\text { Surface } \\
\text { moyenne } \\
\text { en irrigué } \\
\text { (ha) }\end{array}$ & $\begin{array}{l}\text { Effectif de petits } \\
\text { ruminants } \\
\text { reproducteurs }\end{array}$ & $\begin{array}{c}\text { Taux } \\
\text { d'adoption } \\
\text { (nombre } \\
\text { d'adoptants/ } \\
\text { type) }(\%)\end{array}$ \\
\hline $\begin{array}{l}\text { Grands agropasteurs } \\
\text { (EA1) }\end{array}$ & 24 & 34 & 0 & 125 & 54,16 \\
\hline $\begin{array}{l}\text { Agropasteurs } \\
\text { diversifiés (EA2) }\end{array}$ & 53 & 27 & 0 & 64 & 45,28 \\
\hline Petits éleveurs (EA3) & 202 & 15 & 0 & 28 & 18,31 \\
\hline $\begin{array}{l}\text { Multiactifs en irrigué } \\
\text { (El1) }\end{array}$ & 7 & 39 & 5 & 46 & 71,43 \\
\hline $\begin{array}{l}\text { Moyens agriculteurs- } \\
\text { éleveurs (EI2) }\end{array}$ & 11 & 12 & 3 & 30 & 63,64 \\
\hline $\begin{array}{l}\text { Petits agriculteurs- } \\
\text { éleveurs (EI3) }\end{array}$ & 20 & 10 & 1 & 23 & 55 \\
\hline
\end{tabular}

Source des données: « Maghreb \& Mashreq Project », ICARDA, 2002 ; Projet Femise/lcarda, 2004.

pour les éleveurs qui ont déjà une plantation de cactus inerme. Pour comprendre cet écart entre adoption réelle et volonté d'adoption, il faut alors s'intéresser au mode de diffusion de la technologie et plus encore aux relations passées et présentes entre éleveurs et agents du développement. En fait, nombre d'éleveurs ont déclaré être prêts à adopter uniquement si les agents du développement viennent leur présenter la technologie. Certains déclarent n'avoir jamais eu

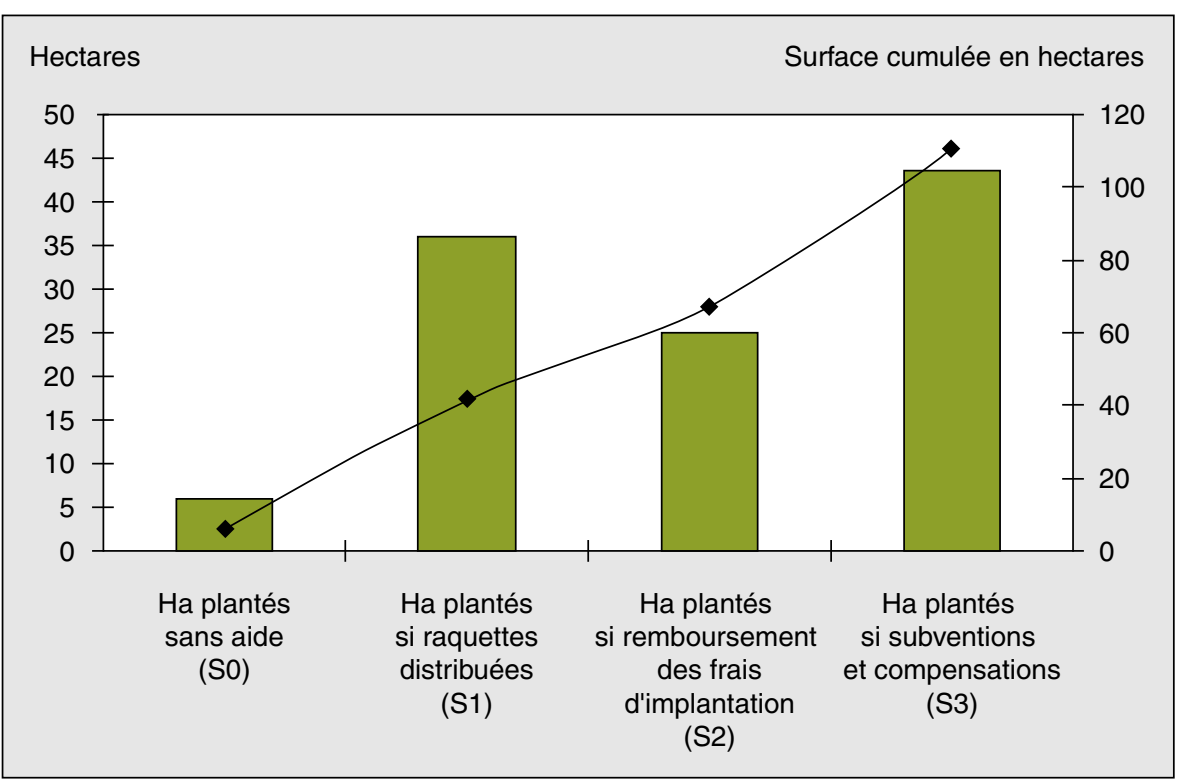

Figure 2. Déclaration d'intention de plantation de cactus inerme intercalaire : surface cumulative de cactus inerme plantée en fonction du niveau de soutien public dans la communauté tunisienne (Zoghmar) (en hectares).

Figure 2. Willingness to use the spineless cactus technology: cumulative area of cactus plantation for different levels of public support in the Zoghmar community (Tunisia) (in hectares).

Projet SPIA/ICARDA, Enquête auprès de 29 éleveurs, 2004. Source : Alary, 2006. la visite d'un agent. Donc problème de reconnaissance sociale, discrimination ou exclusion ressentie, autant de facteurs qui influencent le processus d'adoption.

A contrario, pour les technologies telles que les blocs alimentaires ou les variétés sélectionnées, l'arrêt des approvisionnements lié à la fin du projet de recherche et de développement qui les a promues a mis fin à la possibilité de les utiliser. Pour ces technologies, les raisons sont donc à rechercher dans l'environnement institutionnel : effet de désengagement de l'État, imperfection des marchés, mais aussi démantèlement des dynamiques collectives au sein des communautés.

Ainsi, le processus d'adoption s'inscrit, d'une part dans un système beaucoup plus large (incluant la communauté, le marché et les politiques) et, d'autre part, fait appel à un système de représentation à la fois social et individuel. L'environnement social, économique et politique des producteurs conditionne les facteurs stimulants ou limitants de l'adoption, comme on a pu l'observer en Tunisie. Ces facteurs peuvent être d'ordre physique ou matériel (non-disponibilités sur les marchés, éloignement, manque de moyens, subventions) ou d'ordre social ou immatériel (réseau de connaissance, inégalités communautaires, manque de confiance). Les représentations jouent un rôle encore plus important bien que plus difficile à cerner. Il s'agit d'un mélange de perceptions et d'expérience. 


\section{L'innovation dans le changement social : difficile ajustement}

Ainsi, l'innovation actuelle s'inscrit davantage dans les nouveaux courants ou concepts de la pensée économique rurale, qui donnent la primauté au développement durable, l'équité sociale, l'environnement. On peut citer les techniques de replantation, de lutte contre la désertification, d'économie d'eau. L'innovation elle-même s'appuie le plus souvent sur les pratiques et savoir-faire locaux pour aboutir à un mélange subtil de traditionnel et modernisme. Toutes ces techniques demandent du temps avec des effets à moyen et long termes alors que les exploitations des zones arides, avec la perte des mécanismes traditionnels de lutte contre la sécheresse, se sont inscrites dans des cycles courts de survie. Même hors des périodes de sécheresse, les exploitations sont extrêmement dépendantes des achats d'aliments sur les marchés, ce qui signifie une rentabilité de très court terme.

Le plus souvent ces changements techniques proposés tiennent peu compte du décalage entre les pratiques restées traditionnelles pour des raisons matérielles et les sociétés rurales maghrébines qui sont très extraverties. Les sociétés agropastorales, et plus encore les jeunes générations, sont à court de modernité qu'elles côtoient dans les zones avoisinantes qui bénéficient de l'irrigation. Le modèle irrigué s'est imposé comme modèle de réussite économique et sociale alors que la recherche et le développement proposent des techniques relativement extensives.

Enfin, la plupart des projets de recherche ou de développement s'appuient de nos jours sur des approches participatives, interactives, pour faciliter l'introduction et l'appropriation de l'innovation. Mais paradoxalement à ce mouvement, les sociétés agropastorales deviennent de plus en plus individuelles, mouvement qui s'accentue avec le départ des hommes vers les villes. En outre, la diffusion de l'innovation structure la population entre les bons et mauvais élèves, alors que les rapports sociaux dans la communauté segmentent la population entre les grands et les petits propriétaires, donnant peu de poids à la taille du troupeau. Les résistances à l'innovation tiennent donc aussi aux reconditionnements du statut qu'elles induisent. Tous ces éléments ne doivent pas faire oublier le manque d'un environnement institutionnel minimum pour faciliter le changement induit. Ainsi, le changement souhaité par l'extérieur, notamment dans le nouveau paradigme du développement durable, se heurte à l'histoire du développement qui constitue aujourd'hui le cadrage dans lequel les sociétés évoluent et donc évaluent une innovation. La société agropastorale est très contrastée avec une population jeune à la recherche de survie à court terme et de modernité, généralement tournée vers la ville (en attente de partir), une population vieillissante, peu orientée vers le progrès, et enfin une population féminine à la recherche de techniques peu consommatrices en temps. Il existe donc un besoin de recherche d'un métissage entre ancien et nouveau dans la conception de l'innovation. Et celle-ci doit avoir une utilité sans soumettre les adoptants à des choix socialement, culturellement et économiquement impossibles.

\section{Conclusion}

Les sociétés agropastorales du Maghreb ont connu de profonds changements au cours du $\mathrm{Xx}^{\mathrm{e}}$ siècle, largement induits par les politiques de sédentarisation imposées soit par la force soit par des incitations. Mais cette histoire passée, en structurant la population entre grands et petits, influence toujours les décisions et les pratiques actuelles comme les relations intracommunautaires, qui peuvent faciliter ou freiner le changement induit comme l'introduction d'une technologie. Ainsi les raisons de l'adoption sont bien souvent à rechercher dans l'environnement politique et économique des acteurs, mais aussi l'histoire de ces politiques qui a façonné le milieu, y compris les hommes dans leurs perceptions des interventions et des intentions des décideurs politiques.

Dès lors, le changement spontané est-il du ressort d'une politique de correction (Courade, 2005) ? Malgré tous les traumatismes que les agropasteurs ont connus et les départs (migrations), les éleveurs gardent " confiance". Le changement technique a lieu mais il n'est pas seulement ou nécessairement induit ou lié par les innovations technologiques. Ainsi, suite à la dernière grande sécheresse (1998-2002), se sont développés de façon quasiment spontanée des ateliers d'engraissement rapide des agneaux achetés dans les régions plus favorables afin de profiter de la flambée des prix des agneaux pour les fêtes de Ramadan et l'Aïd-El-Kébir. Donc les milieux arides recèlent d'un potentiel de richesse non négligeable mais bien souvent méconnu ou sous-estimé.

\section{Remerciements}

Cette réflexion sur la dynamique des systèmes agropastoraux s'appuie largement sur les acquis du projet de recherche "The Mashreq/Maghreb Project ", coordonné par l'International Center for Agricultural Research in the Dry Areas (ICARDA) et l'International Food Policy Research Institute (IFPRI) et financé par le Fonds international de développement agricole (Fida), le Fonds arabe pour le développement économique etsocial (Fades), le Centre de recherche pour le développement international (CRDI), le Collective Action and Property Right Initiative (CAPRI), et du projet Femise II (Forum euroméditerranéen des instituts économiques) financé par la commission européenne. Que ces instituts soient remerciés pour leur soutien ainsi que les équipes des programmes nationaux d'Algérie, du Maroc et de Tunisie.

\section{Références}

Abaad A, Bédrani S, Bourbouze A, Chiche J. Les politiques agricoles et la dynamique des systèmes agropastoraux au Maghreb. Options Méditerranéennes Sér B 1995 ; No14. [Online] http ://ressources.ciheam.org/om/pdf/b14/Cl96 0048.pdf.

Alary V, El Mourid M. Contribution de la recherche agricole aux PMEAs des zones arides: de l'intensification au développement durable. In : Alary V, El Mourid M, eds. Éléments de politiques pour faciliter l'accès des PMEAs en zones arides dans les pays de I'UMA aux technologies adaptées: bilan sur les aux technologies adaptees: bilan sur les acquis en matiere d'options technologiques et leurs impacts sur les PMEAs en zones arides face à la globalisation. Tunis: FAO, Bureau sous-régional pour I'Afrique du Nord; International Center for Agricultural Research in the Dry Areas-North Africa Regional Program (ICARDA-NARP), 2002.

Alary V, EI Mourid M. Les politiques alimentaires au Maghreb et leurs conséquences sur les sociétés agro-pastorales. Rev Tiers Monde 2005 ; XLVI : 785-810.

Alary V. Des processus d'adoption de l'innovation dans les zones vulnérables - Exemple d'un projet de recherche et développement au projet de recherche et developpement au Maghreb. Revue

Belhedi A. Le découpage administratif en Tunisie. Revue de Géographie du Maroc 1989 13 (série 2) : 3-25. 
Bourbouze A, Gibon A. Ressources individuelles ou ressources collectives? L'impact du statut des ressources sur la gestion des systèmes d'élevage des régions du pourtour méditerranéen. Options Méditerranéenne Sér A 1995 $32: 289-309$.

Chauveau JP. Le modèle sociologique des sociétés paysannes et l'innovation. In Chauveau JP, Cormier-Salem MC, Mollard E, eds. L'innovation en agriculture. Questions de méthodes et terrains d'observation. Collection "A travers champs". Paris: IRD éditions, 1999.

Courade G. Agropasteurs et marchés en zone aride (Maghreb): Quelles innovations? In : Mekersi S, Alary V, Cherfaoui ML, eds. Les obstacles aux transferts technologiques dans les petites et moyennes exploitations agricoles des zones arides et semi arides du Maghreb. Alger : Institut national de la recherche agronomique d'Algérie (Inraa); Forum euroméditerranéen des instituts économiques (Femise) ; International Center for Agricultura Research in the Dry Areas (ICARDA), 2005.

Elloumi M. Du développement agricole au développement rural. Le cas des zones semiarides en Tunisie. Economie Rurale $1993 ; 213$ 18-23.
Elloumi M, Selmi S, Bensalem H, et al. Adoption and Impact studies in Tunisia. In: Shideed $\mathrm{KH}$, El Mourid M, eds. Adoption and impact assessment of improved technologies in crop livestock production systems in the WANA region. The development of integrated crop/livestock production in low rainfall areas of Mashreq and Maghreb regions (Mashreq/Maghreb Project. Aleppo, (Syria): International Center for Agricultural Research in the Dry Areas (ICARDA), 2005.

El Mourid M, El Ouali A, Ambri M, El Oumri M Goebel W. Caractérisation agro-écologique: outils de gestion et d'aide à la décision en agriculture aléatoire. Al Awania 1996; 92 41-51.

El Mourid M, Karrou M, El Gharous M. La recherche en aridoculture respectueuse de I'environnement. Al Awania 1996 ; 92 : 69-82.

Guillermo Y. Le développement pastoral en Algérie: dirigisme ou laisser-faire? Cah Sc Hum $1990 ; 26: 155-71$.

Lesourd M. L'archipel rural africain en mouvement. In : Gastellu JM, ed. La ruralité dans les pays du Sud à la fin du vingtième siècle. Paris Orstom éditions, 1997.
Nefzaoui A, Elloumi M, Nasr N, et al. Rangeland management options and individual and community strategies of agropastoralists in central and Southern Tunisia. In : Ngaido T, McCarthy N, Di Gregorio M, eds. International Conference on policy and institutional options for the management of rangelands in dry areas. Workshop summary paper. CAPRi Working Paper $\mathrm{N}^{\circ} 23$. Washington (DC) : International Food Policy Research Institute, 2002.

Requier-Desjardins D. L'économie des organisations et l'analyse du comportement des unités domestiques en Afrique subsaharienne. Economie appliquée 1994 ; XLVI : 105-31.

Sandron F. Déterminants des migrations en zone montagneuse forestière tunisienne. In : Gastellu JM, ed. La ruralité dans les pays du Sud à la fin du vingtième siècle. Paris : Orstom éditions, 1997.

Shideed KH, Khatib KK. Monitoring the adoption of feed bolck technologies. In: Shi deed $\mathrm{KH}$, El Mourid M, eds. Adoption and impact assessment of improved technologies in crop livestock production systems in the WANA region. The development of integrated crop/livestock production in low rainfall areas of Mashreq and Maghreb regions (Mashreq/ Maghreb Project). Aleppo (Syria): International Center for Agricultural Research in the Dry Areas (ICARDA), 2005. 